\title{
CLONORCHIS SINENSIS INFECTION AND INCREASING RISK OF CHOLANGIOCARCINOMA IN THE REPUBLIC OF KOREA
}

\begin{abstract}
MIN KYUNG LIM, YOUNG-HEE JU, SILVIA FRANCESCHI, JIN-KYOUNG OH, HYUN-JOO KONG, SEUNG-SIK HWANG, SUE-KYUNG PARK, SUNG-IL CHO, WOON-MOK SOHN, DONG-IL KIM, KEUN-YOUNG YOO, SUNG-TAE HONG, AND HAI-RIM SHIN*

Research Institute for National Cancer Control and Evaluation, National Cancer Center, Goyang, Republic of Korea; Infections and Cancer Epidemiology Group, International Agency for Research on Cancer, Lyon, France; Department of Preventive Medicine, Konkuk University College of Medicine, Chungju, Republic of Korea; Seoul National University Graduate School of Public Health and Institute of Health and Environment, Seoul, Republic of Korea; Department of Parasitology and Institute of Health Sciences, Gyengsang National University College of Medicine, Jinju, Republic of Korea; Department of Occupational Medicine, Kangbuk Samsung Hospital and Sungkunkwan University, Suwon, Republic of Korea; Department of Preventive Medicine, Seoul National University College of Medicine, Seoul, Republic of Korea; Department of Parasitology and Tropical Medicine, Seoul National University College of Medicine, Seoul, Republic of Korea
\end{abstract}

\begin{abstract}
To evaluate the prevalence and risk factors of Clonorchis sinensis infection, an epidemiologic survey was performed (3,169 residents; age range: $30-87$ years; mean age $=59 ; 1,973$ women $)$ in three areas with different mortality rates of cholangiocarcinoma. C. sinensis prevalence was $2.1 \%$ in Chuncheon, $7.8 \%$ in Chungju, and $31.3 \%$ in Haman, where cholangiocarcinoma incidence rate was $0.3,1.8$, and 5.5 per 100,000 persons, respectively. Of the four major risk factors for $C$. sinensis (male sex, alcohol consumption, raw freshwater fish intake, and area of residence), area was the strongest. Efforts to discourage raw freshwater fish intake, treat egg carriers, and protect water sources from $C$. sinensis contamination should be strengthened in the Republic of Korea.
\end{abstract}

\section{INTRODUCTION}

Clonorchis sinensis, the oriental liver fluke, causes clonorchiasis, which is characterized by hyperplasias and metaplasias in the intrahepatic bile duct epithelium. Clonorchiasis is endemic predominantly in Asian countries, such as China, Taiwan, Vietnam, and the Republic of Korea. ${ }^{1,2}$ Most infected individuals have few symptoms, but some of them suffer from abdominal pain and other symptoms from complications. The complications of chronic clonorchiasis include intrahepatic stones, biliary cirrhosis, pyogenic cholangitis, and cholangiocarcinoma. ${ }^{3-5}$ Indeed, the geographic distribution of cholangiocarcinoma worldwide coincides with the distribution of $C$. sinensis and Opisthorchis viverrini, another liver fluke of the Opisthorchidae family. ${ }^{3-5}$ In Korea, which has been known as an endemic area of $C$. sinensis infection, mortality of cholangiocarcinoma accounts for $5.8 \%$ of primary liver cancer. The age-standardized mortality rate is $1.2 /$ 100,000 persons $(1.8 / 100,000$ men and $0.8 / 100,000$ women), but the standardized mortality ratio of the disease varies 4-fold within the country, ranging from $50.6 \%$ to $218.3 \%$ among provinces. ${ }^{6}$

Clonorchis sinensis is transmitted through snails to freshwater fish and then to humans and other fish-eating mammals. Humans are infected by eating raw freshwater fish, and therefore it has been prevalent along rivers in Korea for a long time. To elucidate the relationships between the prevalence of and risk factors for parasite infection, we evaluated the presence of $C$. sinensis eggs in the stool samples and life habits of individuals from three different areas of Korea, each showing different cholangiocarcinoma mortality. ${ }^{6}$

\section{MATERIALS AND METHODS}

This study is based on the cross-sectional findings of a cohort study carried out by the Korean National Cancer Center.

* Address correspondence to Hai-Rim Shin, Research Institute for National Cancer Control and Evaluation, National Cancer Center, 809 Madu-dong, Ilsandong-gu, Goyang, Gyeonggi 411-769, Republic of Korea. E-mail: hrshin@ncc.re.kr
The cohort study included men and women $\geq 30$ years of age who participated in cancer screening surveys and are linked to the Korean Multi-center Cancer Cohort. ${ }^{7}$ Three areas, which are located in vicinity of river, are the subject of this report (Figure 1): Chuncheon-si (city) in Gangwon-do (province) (age-standardized mortality rates of cholangiocarcinoma: 0.9/ 100,000 men and 0.5/100,000 women), Chungju-si in Chungcheongbuk-do (rates of 2.2 and 0.6 per 100,000 , respectively), and Haman-gun (county) in Gyeongsangnam-do (rates of 4.4 and 1.5 per 100,000 , respectively). ${ }^{6}$ A total of 3,169 subjects (age range: $30-87$; mean age $=59$ years) were recruited between 2000 and 2004, including 659 from Chuncheon, 568 from Chungju, and 1,942 from Haman. Each participant was interviewed by a trained interviewer, and blood and stool samples were collected for laboratory examination.

Informed consent was obtained from all participants, and the study protocol was approved by the National Cancer Center of Korea. The questionnaire included information on education, tobacco smoking, alcohol consumption, history of $C$. sinensis infection, and raw freshwater fish intake. To determine hepatitis B and C virus infection, enzyme linked immunosorbent assays (ELISA; HBsAg [V2] and HCV [V3], AxSYM System; Abbott, Abbott Park, IL) were used. Samples were tested for seropositivity to hepatitis B surface antigen (HBsAg) and anti-HCV antibodies (anti-HCV). Stool samples were examined by means of the formalin-ether sedimentation method to identify the presence of $C$. sinensis eggs.

Unconditional multiple logistic regression was used to calculate odds ratios (ORs) and corresponding 95\% confidence intervals (CIs) of being C. sinensis egg-positive according to area and various characteristics.

\section{RESULTS}

Positivity for C. sinensis eggs was $2.1 \%$ in Chuncheon, $7.8 \%$ in Chungju, and $31.3 \%$ in Haman. C. sinensis infection was found more often in men than women in all areas, but the difference was especially marked in Chungju (OR in men versus women $=9.4 ; 95 \% \mathrm{CI}$ : 4.2-21.0). Positivity declined 


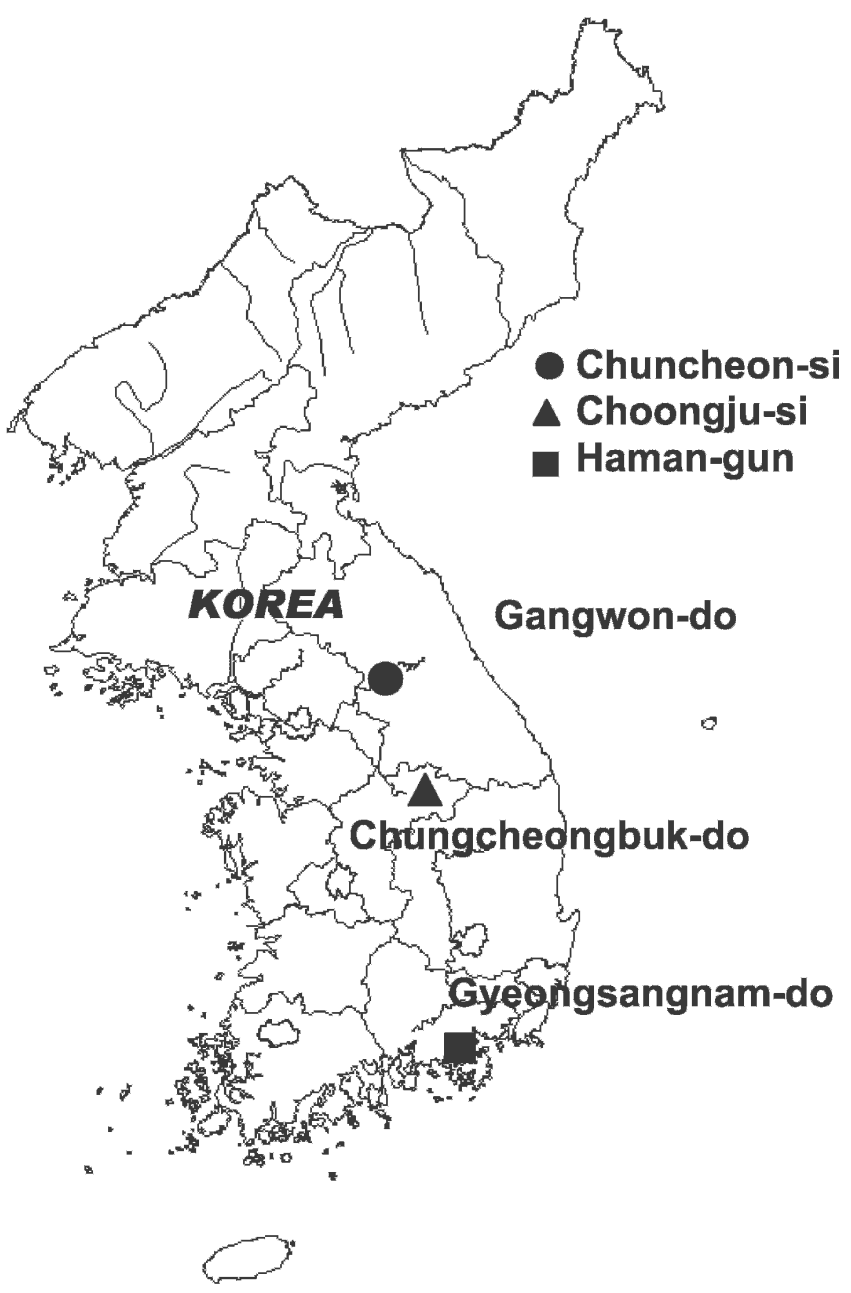

FIGURE 1. Location of participating centers in the study on $C$. sinensis, Republic of Korea, 2000-2004.

significantly with age in Chungju and in Haman, but not in Chuncheon. Alcohol consumption was significantly associated with $C$. sinensis positivity in Haman but not elsewhere (Table 1). History of $C$. sinensis infection was positively associated with the presence of eggs in Chuncheon $(\mathrm{OR}=16.6)$ but negatively associated in Haman $(\mathrm{OR}=0.6)$. The $\mathrm{OR}$ for C. sinensis associated with raw freshwater fish intake was significantly elevated in Chungju (OR $=2.9$; $95 \%$ CI: $1.5-$ 5.7) but not in the other areas. Educational level, tobacco smoking, HbsAg, and anti-HCV positivity were not related to egg positivity of $C$. sinensis (Table 1).

Table 2 shows ORs of $C$. sinensis positivity in the entire study population from a multivariate model including gender, alcohol consumption, raw freshwater fish intake, and area of residency. Significantly elevated ORs were found for all four characteristics considered, but the area of residence showed by far the largest influence (OR for Haman versus Chuncheon $=14.1 ; 95 \%$ CI: 7.9-25.0, Table 2).

\section{DISCUSSION}

Clonorchis sinensis was defined as a risk factor (carcinogen group 2A) for hepatobiliary cancer by International Agency for Research on Cancer (IARC). ${ }^{4}$ However, there are few studies to support this considerable relationship even though prevalence of clonorchiasis in human being is increasing or still prevalent in various parts of endemic area. ${ }^{1,8}$ In this aspect, this study suggests the timely and necessary results for correlation between the endemicity of $C$. sinensis and the risk of cholangiocarcinoma. The differences in prevalence of $C$. sinensis infection corresponded with the differences in mortality and incidence of cholangiocarcinoma among subject areas. Basically, the mortality and incidence come from the national mortality data (1999-2001) of Korean National Statistical Office and nationwide incidence data (1991-2001) from the Ministry of Health and Welfare, respectively, which is based on the Cause of Death Statistics and Korea Central Cancer Registry database. ${ }^{6,9}$ To get subject area specific mortality and incidence, we analyzed the raw data of each database and produced the mortality and incidence rate for cholangiocarcinoma (i.e., C22.1 in the 10th International Classification of Disease) standardized for age with world population. The age-standardized mortality rate of cholangiocarcinoma in Chuncheon, which was a non-endemic area of $C$. sinensis infection with $2.1 \%$ egg positive rate, was $0.2 /$ 100,000 persons. $^{6}$ In that non-endemic area, the carcinoma mortality and the egg positive rate were lower than average in Korea (i.e., age-standardized mortality rate: $1.2 / 100,000$ persons, egg positive rate: $2.9 \%) .{ }^{6,9}$ However, the mortality of cholangiocarcinoma was 1.1 and 2.6 per 100,000 persons in Chungju and Haman, where the prevalence of $C$. sinensis infection were at the low and moderate endemic level, respectively. ${ }^{6,8}$ Similar with the mortality, incidence of cholangiocarcinoma also correlated with the prevalence of $C$. sinensis infection with Haman showing relatively high incidence rate (i.e., age-standardized incidence rate of 5.5/100,000 persons) compared with the other two areas (i.e., age-standardized incidence rate per 100,000 persons: 1.8 for Chungju and 0.3 for Chuncheon). ${ }^{9}$ In addition, the proportion of cholangiocarcinoma among incident cases of liver cancer (C22) varied with subject areas (i.e., $13.2 \%$ in Haman, $8.9 \%$ in Chungju, and $1.4 \%$ in Chuncheon). ${ }^{9}$ These epidemiologic data support that the increasing risk of mortality and incidence of cholangiocarcinoma in some parts of country is possibly caused by the high endemicity of $C$. sinensis infection in these areas.

In Korea, after the introduction of chemotherapy for $C$. sinensis using praziquantel in the 1980s, successful control of clonorchiasis was expected. ${ }^{5}$ However, our study shows that C. sinensis infection is still common and endemic in some parts of Korea. Indeed, the nationwide surveys of intestinal parasite infections revealed no decrease in the average prevalence of $C$. sinensis despite praziquantel medication (i.e., $2.6 \%$ in 1981 and $2.9 \%$ in 2004). ${ }^{8}$ The estimate of infected people nationwide was 1.3 million in 2004, and therefore, clonorchiasis is seriously threatening to human health in Korea, especially in endemic areas. ${ }^{8}$ Haman is a rural area located near the Nakdong river, and residents of Haman are mainly farmers and/or fishermen. River or reservoirs in vicinity of Haman have been shown to have a high density of the fish hosts of $C$. sinensis with fish showing high load of metacercariae and residents of the endemic areas prefer eating raw fish. ${ }^{10}$ Approximately $2 \%$ of the population is positive for clonorchiasis throughout the country, and even in nonendemic areas, like Chuncheon of this study, and the prevalence is regarded as its infection status caused by eating raw fish when they visit the endemic area. 
TABLE 1

Odds ratios and corresponding $95 \%$ confidence intervals for the presence of $C$. sinensis eggs in stool by selected characteristics and area, Republic of Korea, 2000-2004

\begin{tabular}{|c|c|c|c|c|c|c|c|c|c|}
\hline & \multicolumn{3}{|c|}{ Chuncheon } & \multicolumn{3}{|c|}{ Chungju } & \multicolumn{3}{|c|}{ Haman } \\
\hline & $N$ & No. of positive (\%) & OR $(95 \% \mathrm{CI})^{*}$ & $N$ & No. of positive (\%) & OR $(95 \% \mathrm{CI})^{*}$ & $N$ & No. of positive (\%) & OR $(95 \% \mathrm{CI})^{*}$ \\
\hline \multicolumn{10}{|l|}{ Sex } \\
\hline Female & 470 & $8(1.7)$ & Reference & 351 & $8(2.3)$ & Reference & 1,152 & $319(27.7)$ & Reference \\
\hline Male & 189 & $6(3.2)$ & $1.73(0.59-5.10)$ & 217 & $36(16.6)$ & $9.43(4.24-20.97)$ & 790 & $288(36.5)$ & $1.51(1.25-1.84)$ \\
\hline \multicolumn{10}{|l|}{ Age (years) } \\
\hline$<55$ & 220 & $3(1.4)$ & Reference & 158 & $15(9.5)$ & Reference & 583 & $197(33.8)$ & Reference \\
\hline $55-64$ & 201 & $3(1.5)$ & $1.11(0.22-5.58)$ & 192 & $19(9.9)$ & $0.77(0.36-1.63)$ & 724 & $233(32.2)$ & $0.93(0.74-1.17)$ \\
\hline$\geq 65$ & 238 & $8(3.4)$ & $2.40(0.62-9.19)$ & 218 & $10(4.6)$ & $0.33(0.14-0.77)$ & 635 & $177(27.9)$ & $0.74(0.58-0.95)$ \\
\hline \multicolumn{10}{|c|}{ Education level (years) } \\
\hline 0 & 128 & $4(3.1)$ & Reference & 84 & $3(3.6)$ & Reference & 423 & $128(30.3)$ & Reference \\
\hline $1-6$ & 292 & $4(1.4)$ & $0.50(0.11-2.19)$ & 264 & $18(6.8)$ & $0.82(0.22-3.12)$ & 933 & $282(30.2)$ & $0.83(0.67-1.13)$ \\
\hline$\geq 7$ & 239 & $6(2.5)$ & $1.12(0.24-5.33)$ & 220 & $23(10.5)$ & $0.74(0.19-2.95)$ & 586 & 197 (33.6) & $0.87(0.64-1.18)$ \\
\hline \multicolumn{10}{|c|}{ Smoking status } \\
\hline Never & 463 & $9(1.9)$ & Reference & 381 & $15(3.9)$ & Reference & 1,181 & $333(28.2)$ & Reference \\
\hline Former & 81 & $1(1.2)$ & $0.31(0.03-3.30)$ & 91 & $16(17.6)$ & $1.53(0.59-3.94)$ & 245 & $82(33.5)$ & $1.09(0.75-1.59)$ \\
\hline Current & 95 & $4(4.2)$ & $1.33(0.27-6.67)$ & 92 & $13(14.1)$ & $1.00(0.38-2.62)$ & 515 & $191(37.1)$ & $1.24(0.92-1.69)$ \\
\hline \multicolumn{10}{|c|}{ Alcohol consumption } \\
\hline Never & 354 & $8(2.3)$ & Reference & 309 & $13(4.2)$ & Reference & 1,212 & $321(26.5)$ & Reference \\
\hline$<24 \mathrm{~g} / \mathrm{d}$ & 102 & $2(2.0)$ & $0.67(0.11-3.89)$ & 148 & $17(11.5)$ & $1.48(0.65-3.36)$ & 298 & $114(37.9)$ & $1.59(1.20-2.11)$ \\
\hline$\geq 24 \mathrm{~g} / \mathrm{d}$ & 59 & $3(5.1)$ & $1.31(0.21-8.02)$ & 72 & $10(13.9)$ & $0.97(0.37-2.58)$ & 330 & $135(41.5)$ & $1.81(1.33-2.46)$ \\
\hline \multicolumn{10}{|l|}{ HBsAg } \\
\hline Negative & 635 & $14(2.2)$ & Reference & 552 & $44(8.0)$ & Reference & 1,594 & $509(31.9)$ & Reference \\
\hline Positive & 21 & $0(0.0)$ & - & 14 & $0(0.0)$ & - & 85 & $28(32.9)$ & $1.01(0.63-1.61)$ \\
\hline \multicolumn{10}{|l|}{ Anti-HCV } \\
\hline Negative & 646 & $14(2.2)$ & Reference & 560 & $44(7.9)$ & Reference & 1,650 & $525(31.8)$ & Reference \\
\hline Positive & 10 & $0(0.0)$ & - & 6 & $0(0.0)$ & - & 30 & $13(43.3)$ & $1.54(0.74-3.21)$ \\
\hline \multicolumn{10}{|c|}{ History of $C$. sinensis infection } \\
\hline Never & 553 & $10(1.8)$ & Reference & 548 & $39(7.1)$ & Reference & 1,682 & $542(32.2)$ & Reference \\
\hline Ever & 5 & $1(20.0)$ & $16.57(1.55-176.88)$ & 8 & $2(25.0)$ & $2.18(0.40-11.92)$ & 207 & $50(24.2)$ & $0.57(0.41-0.81)$ \\
\hline \multicolumn{10}{|c|}{ Raw freshwater fish intake } \\
\hline Never & 434 & $8(1.8)$ & Reference & 449 & $21(4.7)$ & Reference & 249 & $70(28.1)$ & Reference \\
\hline Ever & 225 & $6(2.7)$ & $1.50(0.50-4.51)$ & 119 & $23(19.3)$ & $2.92(1.50-5.69)$ & 1693 & $537(31.7)$ & $1.16(0.86-1.56)$ \\
\hline Total & 659 & $14(2.1)$ & - & 568 & $44(7.8)$ & - & 1,942 & $607(31.3)$ & - \\
\hline
\end{tabular}

Area of residence was by far the strongest risk factor for $C$. sinensis in our study. The influence of other personal characteristics or habits was comparatively modest and mainly found in the low-endemic area (i.e., Chungju). This held true for male sex and raw freshwater fish intake, which were substantially more clearly associated with the presence of $C$. sinensis eggs in stool samples in Chungju than in non- or moderate-endemic areas. Interestingly, in Haman, where the vast majority of the study population reported raw freshwater fish intake and the prevalence of eggs was only $50 \%$ higher in men than in women, high alcohol consumption was a better marker of $C$. sinensis infection than raw freshwater fish intake. Indeed, concurrent consumption of alcohol and raw freshwater fish is frequent in Korea.

No correlation emerged, as expected, between mainly foodborne $C$. sinensis infection and markers of infection with hepatitis $\mathrm{B}$ or $\mathrm{C}$ viruses, which are transmitted chiefly from mother to child or through contaminated blood and needles. ${ }^{11}$ However, the negative association between history of $C$. sinensis infection and detection of eggs in Haman was surprising. It is conceivable that previous diagnosis of the parasite led to chemotherapy, possibly resulting in the elimination of the parasite eggs from stool samples. However, exclusion of all individuals with a history of $C$. sinensis infection did not materially modify any of our findings, including the vast variation by area of residence and the apparent de- cline in the detection of eggs in individuals $>54$ years of age. Other studies also reported that $C$. sinensis infection was more commonly found in middle-aged individuals than in the elderly. 5,12,13 This suggests that, in endemic areas of Korea,

TABLE 2

Odds ratios and corresponding 95\% confidence intervals for the presence of $C$. sinensis eggs in stool samples by sex, alcohol consumption, raw freshwater fish intake and area, Republic of Korea, 2000-2004

\begin{tabular}{|c|c|c|c|}
\hline & $N$ & $\begin{array}{c}\text { No. of positive } \\
(\%)\end{array}$ & $\begin{array}{l}\text { Multivariate OR } \\
\quad(95 \% \text { CI })\end{array}$ \\
\hline \multicolumn{4}{|l|}{ Sex } \\
\hline Female & 1,973 & $335(17.0)$ & Reference \\
\hline Male & 1,196 & $330(27.6)$ & $1.32(1.05-1.67)$ \\
\hline \multicolumn{4}{|c|}{ Alcohol consumption } \\
\hline Never & 1,875 & $342(18.2)$ & Reference \\
\hline$<24 \mathrm{~g} / \mathrm{d}$ & 548 & $132(24.1)$ & $1.55(1.19-2.01)$ \\
\hline$\geq 24 \mathrm{~g} / \mathrm{d}$ & 461 & $150(32.5)$ & $1.66(1.24-2.23)$ \\
\hline \multicolumn{4}{|c|}{ Raw freshwater fish intake } \\
\hline Never & 1,132 & $99(8.8)$ & Reference \\
\hline Ever & 2,037 & $566(27.8)$ & $1.50(1.2-1.99)$ \\
\hline \multicolumn{4}{|l|}{ Area } \\
\hline Chuncheon & 659 & $14(2.1)$ & Reference \\
\hline Chungju & 568 & $44(7.8)$ & $3.15(1.66-5.99)$ \\
\hline Haman & 1,942 & $607(31.3)$ & $14.06(7.90-25.00)$ \\
\hline Total & 3,169 & $665(21.0)$ & - \\
\hline
\end{tabular}


the prevalence of $C$. sinensis infection is not declining in subsequent cohorts of adults despite extensive efforts that have been ongoing since the 1970 s to discourage the consumption of raw freshwater fish and to identify and treat infected individuals. $^{12,14}$

A limitation of this study is the impossibility to quantify accurately the amount and frequency of raw freshwater fish intake. However, hundreds of parasites can be harbored within a single fish, and most of the live metacercariae introduced into humans have a successful parasitic life. ${ }^{5}$ Therefore, any amount of intake of contaminated raw freshwater fish could lead to $C$. sinensis infection, although not necessarily to severe complications such as chronic and heavy clonorchiasis. The type of population involved (voluntary participants in a cancer screening survey) is unlikely to have distorted our findings, because the study design was the same in the three study areas, and education level seemed unrelated to $C$. sinensis infection. ${ }^{7}$

In conclusion, great efforts are still required for control of C. sinensis in endemic areas of Korea to reduce the incidence clonorchiasis and cholangiocarcinoma. In addition to the elimination of the consumption of raw, undercooked, or fresh pickled fish and prolonged or repeated chemotherapy of infected individuals, the protection of fish ponds and aquaculture systems from contamination with feces from people and animals should be strengthened for prevention of its infection. ${ }^{15,16}$ Furthermore, longitudinal studies to identify the relationship between endemicity of $C$. sinensis infection and risk of cholangiocarcinoma should be done in the endemic area of Korea.

Received January 2, 2006. Accepted for publication March 14, 2006.

Acknowledgments: Min Kyung Lim and Young-Hee Ju share first authorship of this manuscript.

Financial support: This study was supported by National Cancer Center Grant 0110250 . The work reported in this paper was undertaken during Dr. M. K. Lim's tenure as a Postdoctoral Fellowship awardee from the International Agency for Research on Cancer.

Authors' addresses: Min Kyung Lim, Young-Hee Ju, Jin-Kyoung Oh, Hyun-Joo Kong, Seung-Sik Hwang, and Hai-Rim Shin, Research Institute for National Cancer Control \& Evaluation, National Cancer Center, 809 Madu-dong, Ilsandong-gu, Goyang, Gyeonggi, 411-769, Republic of Korea. Silvia Franceschi, Infections and Cancer Epidemiology Group, International Agency for Research on Cancer, 150 cours Albert Thomas, 69372 Lyon cedex 08, France. Sue-Kyung Park, Department of Preventive Medicine, Konkuk University College of Medicine, 322 Danwol-dong, Chungju, Chungbuk, 380-701, Republic of Korea. Sung-Il Cho, Graduate School of Public Health and Institute of Health and Environment, Seoul National University, 28 Yeongeon-dong, Jongno-gu, Seoul 110-799, Republic of Korea. Woon-Mok Sohn, Department of Parasitology and Institute of Health Sciences, Gyeongsang National University College of Medicine, 92 Chiram-dong, Jinju, Gyeongnam, Republic of Korea. Dong-Il Kim, Department of Occupational Medicine, Kangbuk Samsung Hospital and Sungkunkwan University, 108 Pyoung-dong, Jongno-gu, Seoul, Republic of Korea. Keun-Young Yoo, Department of Preventive Medicine, Seoul National University College of Medicine, 28 Yeongeon-dong, Jongno-gu, Seoul 110-799, Republic of Korea. Sung-Tae Hong, Department of Parasitology and Tropical Medi- cine, Seoul National University College of Medicine, 28 Yeongeondong, Jongno-gu, Seoul 110-799, Republic of Korea.

Reprint requests: Dr. Hai-Rim Shin, Research Institute for National Cancer Control and Evaluation, National Cancer Center, 809 Madudong, Ilsandong-gu, Goyang, Gyeonggi 411-769, Republic of Korea. E-mail: hrshin@ncc.re.kr.

\section{REFERENCES}

1. Lun ZR, Gasser RB, Lai DH, Li AX, Zhu XQ, Yu XB, Fang YY, 2005. Clonorchiasis: a key foodborne zoonosis in China. Lancet Infect Dis 5: 31-41.

2. Yeh TC, Lin PR, Chen ER, Shaio MF, 2001. Current status of human parasitic infections in Taiwan. $J$ Microbiol Immunol Infect 34: 155-160.

3. Srivatanakul P, Sriplung H, Deerasamee S, 2004. Epidemiology of liver cancer: an overview. Asian Pac J Cancer Prev 5: 118125.

4. International Agency for Research on Cancer (IARC), 1993. Monographs on the Evaluation of Carcinogenic Risks to Humans. Volume 61: Schistosomes, Liver Flukes and Helicobacter pylori. Lyon, France: International Agency for Research on Cancer (IARC) Press.

5. Hong ST, 2003. Clonorchis sinensis. Miliotis MD, Bier JW, eds. International Handbook of Foodborne Pathogens. New York: Marcel Dekker, 581-592.

6. Korean National Statistical Office, 1999-2001. Annual Report on the Cause of Death Statistics based on Vital Registration. Seoul, Korea: Korean National Statistical Office.

7. Yoo KY, Shin HR, Chang SH, Lee KS, Park SK, Kang D, Lee DH, 2002. Korean multi-center cancer cohort study including a biological materials bank (KMCC-I). Asian Pac J Cancer Prev 3: 85-92.

8. Korean Ministry of Health and Welfare, Korean Association of Health Promotion, 2004. Prevalence of Intestinal Parasitic Infection in Korea-The $7^{\text {th }}$ Report. Seoul: Korean Ministry of Health and Welfare, Korean Association of Health Promotion, 61-66.

9. Shin HR, Won YJ, Jung KW, Kong HJ, Yim SH, Lee JK, Noh HI, Lee JK, Pisani P, Park JG, 2005. Nationwide cancer incidence in Korea, 1999-2001: First resulting using the national cancer incidence database. Cancer Res Treat 37: 325-331.

10. Rim HJ, Kim KH, Joo KH, Kim SJ, Eom KS, Chung MS, 1996. The infestation states and changing patterns of human infecting metacercariae in freshwater fish in Kyongsang-do and Kyonggi-do, Korea. Korean J Parasitol 34: 95-105.

11. Shin HR, Kim JY, Kim JI, Lee DH, Yoo KY, Lee DS, Franceschi $S, 2002$. Hepatitis B and C virus prevalence in a rural area of South Korea: the role of acupuncture. Br J Cancer 87: 314-318.

12. Kim BJ, Ock MS, Kim IS, Yeo UB, 2002. Infection status of Clonorchis sinensis in residents of Hamyang-gun, Gyeongsangnam-do, Korea. Korean J Parasitol 40: 191-193.

13. Joo CY, Chung MS, Kim SJ, Kang CM, 1997. Changing patterns of Clonorchis sinensis infections in Kyongbuk, Korea. Korean J Parasitol 35: 155-164.

14. Ju YH, Oh JK, Kong HJ, Sohn WM, Kim JI, Jung KY, Kim YG, Shin HR, 2005. Epidemiologic study of Clonorchis sinensis infestation in a rural area of Kyongsangnam-do, Korea. J Prev Med Public Health 38: 425-430.

15. Hong ST, Rim HJ, Min DY, Li X, Xu J, Feng Z, Lee SH, 2001. Control of clonorchiasis by repeated treatments with praziquantel. Korean J Parasitol 39: 285-292.

16. Garrett ES, dos Santos CL, Jahncke ML, 1997. Public, animal, and environmental health implications of aquaculture. Emerg Infect Dis 3: 453-457. 